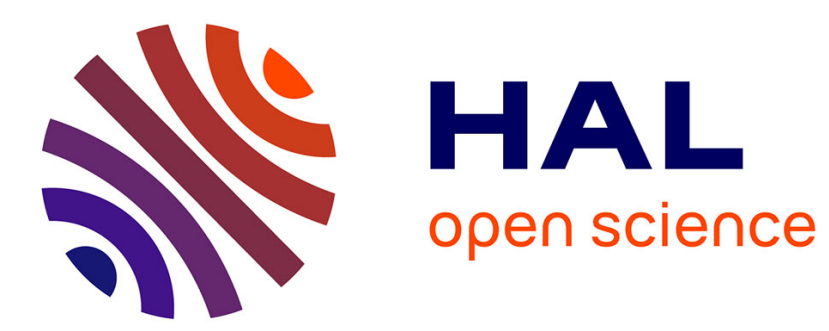

\title{
Pratiques de recherche documentaire et réussite universitaire des étudiants de première année
}

Cathy Perret

\section{To cite this version:}

Cathy Perret. Pratiques de recherche documentaire et réussite universitaire des étudiants de première année. Carrefours de l'éducation, 2013, 35, pp.197-215. halshs-00837782

\section{HAL Id: halshs-00837782 \\ https://shs.hal.science/halshs-00837782}

Submitted on 29 May 2017

HAL is a multi-disciplinary open access archive for the deposit and dissemination of scientific research documents, whether they are published or not. The documents may come from teaching and research institutions in France or abroad, or from public or private research centers.
L'archive ouverte pluridisciplinaire HAL, est destinée au dépôt et à la diffusion de documents scientifiques de niveau recherche, publiés ou non, émanant des établissements d'enseignement et de recherche français ou étrangers, des laboratoires publics ou privés. 


\section{Cathy Perret}

Cathy.perret@u-bourgogne.fr

\section{PRATIQUES DE RECHERCHE DOCUMENTAIRE ET RÉUSSITE UNIVERSITAIRE DES ÉTUDIANTS DE PREMIÈRE ANNÉE}

a réussite à l'université, résultat d'un processus d'affiliation (Coulon, 1997) ou d'intégration (Dubet, 1994), repose en partie sur l'apprentis-

sage de nouvelles normes et formes de travail largement implicites lors de l'entrée à l'université (Coulon, 1997). La transition du secondaire vers le supérieur se fait ainsi dans « un flou pédagogique » (Oberti, 1995). Et ce flou est entretenu par les enseignants peu explicites quant aux pratiques de travail à mettre en œuvre pour réussir (Boyer et Coridian, 2002). La capacité à se documenter de manière autonome est primordiale pour réussir (Coulon, 1999). Et elle est présentée par les enseignants comme une condition pour réussir, (même si les scientifiques insistent peu auprès des étudiants) (Boyer et Coridian, 2002). Une telle capacité renvoie aux compétences des étudiants en termes de méthodologie documentaire traditionnellement considérées comme faibles à l'entrée à l'université (Mittermeyer et Quirion, 2003; Thirion et Pochet, 2008).

L'arrivée de nouveaux publics étudiants (Galland, 2009) pose désormais de nouvelles questions aux enseignants comme aux professionnels de la documentation (Poissenot, 2004), perceptibles à travers les enquêtes récentes sur les bibliothèques (cf. recensement de Renoult, 2006) ; les étudiants ayant par exemple un autre rapport à la lecture (Coulangeon, 2009). En outre, avec l'avènement des technologies de l'information et de la communication (TIC), les pratiques étudiantes se sont modifiées. Début 2000, une enquête menée auprès de 2000 étudiants américains a ainsi montré leurs avis positifs sur l'usage d'Internet pour leurs études (Jones, 2002). Si des débats se sont organisés sur l'utilisation 
d'Internet par les étudiants (Bertrand, 2002), si les recherches et récits d'expériences sur les dispositifs pédagogiques liés aux TIC sont nombreux (cf. Revue Internationale des Technologies en Pédagogie Universitaire) si des études existent sur les pratiques d'Internet par les étudiants (Frenette et Johnsson-Smaragdi, 2004 ; Lima et Granon, 2007), peu s’intéressent à leurs liens avec les méthodes de travail. L'usage spécifique d'Internet pour les études est plus difficile que pour un usage ordinaire (Faurie et Van de Leemput, 2007). Et les pratiques étudiantes sont marquées par des recours différenciés à Internet liés au sexe, aux ressources et au niveau d'études (Faurie et al. , 2004), au sentiment d'efficacité sur les TIC (Faurie et Van de Leemput, 2007). Toutefois, I'utilisation d'Internet semble peu favoriser les compétences informationnelles permettant d'atteindre les compétences requises par le métier d'étudiant (Thirion et Pochet, 2008).

Notre objectif est d'identifier l'existence ou non de pratiques différenciées et leurs effets possibles sur la réussite universitaire. Dans la mesure où ces pratiques sont susceptibles d'être conditionnées par les contextes locaux (les travaux de l'Observatoire de la Vie Étudiante le rappellent régulièrement), elles sont étudiées à partir d'une enquête menée dans une seule formation de $1^{\text {re }}$ année de l'université de Bourgogne (UB) par le Centre d’innovation pédagogique et d'évaluation (CIPE). Après la présentation du contexte de la recherche, cet article exposera des premiers éléments concernant la recherche documentaire. Seront ensuite examinées les différentes pratiques de recherches documentaires coexistantes, puis recherchés leurs effets possibles sur la réussite lors de l'entrée à l'université.'

\section{PRÉSENTATION DE LA RECHERCHE EMPIRIQUE}

Cette recherche utilise une enquête réalisée auprès d'étudiants de $1^{\text {re }}$ année de licence langues littératures et civilisations étrangères (LLCE) de l'UB en décembre 2007 et sur des données individuelles administratives (inscriptions et examens via Apogée). 174 étudiants, soit 62 \% des inscrits pédagogiques, ont répondu au questionnaire distribué lors d'un cours magistral par le responsable de la licence. Des différences significatives existent entre la population ayant ou non participé : une surreprésentation des filles (83\% contre $69 \%$ pour les non répondants) et une forte surreprésentation des étudiants bacheliers de l'année 2007 (72 \% contre 46 \% pour les non répondants) et des étudiants titulaires 
d'un baccalauréat général (L, ES, S) (94\% contre $79 \%)$. Au regard des résultats aux examens, les répondants sont très spécifiques : sont sous-représentés les étudiants défaillants comme les ajournés (moyenne générale inférieure à 10/20) aux épreuves du premier semestre, du second semestre et sur l'année, alors que ceux ayant réussi leurs examens sont toujours surreprésentés.

L'enquête s'intègre dans un dispositif général d'évaluation d'une expérience de formation à la recherche documentaire menée à l'UB en 2007-08 par le Service commun de documentation (SCD) (cf. encadré). Le questionnaire est composé de quatre parties (cf. annexe). La première comporte des questions sur l'étudiant: date de naissance, lycée d'origine, filière, informations sur l'inscription, fréquentation du CDI (Centre de documentation et d'information) au lycée, formation documentaire au lycée, et accès à un ordinateur. La deuxième partie s'intéresse aux méthodes de travail : fréquentation de la bibliothèque, supports de travail préférés, travail des cours, préparation des devoirs et expériences sur les cours en ligne. La troisième partie est consacrée aux méthodes documentaires: définitions, contextes et méthodes des recherches documentaires, outils utilisés, critères de qualité sur Internet, recueil d’avis. Enfin, la dernière partie est dédiée principalement à la perception de la formation documentaire réalisée par le SCD. D'autres questions sur les services offerts par le SCD, la consultation du site du SCD ainsi que sur des compétences étudiantes sont incluses.

Dispositif d'évaluation réalisé par le Centre d'innovation et d'évaluation pédagogique (CIPE) pour l'enseignement de la recherche documentaire

\section{La première vague du premier semestre 2007-2008}

- Une enquête auprès des étudiants (une semaine avant le second CM de méthodologie documentaire) dont les principaux objectifs sont : 1) connaître les méthodes documentaires des étudiants de $1^{\text {re }}$ année ; 2 ) estimer l'intérêt des étudiants pour ce cours (en termes de méthodes de travail, apprentissages, etc.) ; 3) recueillir les points de vue des étudiants sur le premier CM dispensé durant la semaine d'intégration; et 4) mesurer les acquis des étudiants en termes de méthodologie documentaire pour appréhender les connaissances des étudiants deux mois après le premier CM de méthodologie documentaire.

- À l'issue du premier CM dispensé par trois formateurs du SCD, ces derniers ont exprimé par écrit leurs sentiments et leurs analyses concernant cet enseignement. 


\section{La seconde vague à la fin de l'année universitaire}

- Une enquête auprès des étudiants avant ou pendant les examens du second semestre afin de 1) mesurer l'utilisation de l'outil d'enseignement en ligne de la recherche documentaire construit en 2007-2008; 2) recueillir des avis des étudiants sur cet outil (aspects techniques et pédagogiques) 3) mesurer les acquis en termes de méthodologie documentaire.

- Une enquête auprès des enseignants volontaires pour expérimenter ce nouvel enseignement afin de 1) recueillir leurs avis sur le dispositif d'enseignement proposé et les aménagements souhaitables; 2) connaître leurs opinions quant à son utilisation par les étudiants ;

- Un recueil d'informations a été fait pour connaître le nombre de connexions étudiantes (soumises à identification).

La construction de ce dispositif en deux temps (décembre et printemps) résulte de différents compromis : ne pas multiplier les enquêtes et favoriser la participation étudiante. C'est pourquoi il n'y a pas eu d'enquêtes de satisfaction sur les CM du SCD.

\section{PREMIERS CONSTATS SUR LA RECHERCHE DOCUMENTAIRE}

L'enquête permet différents constats : Comment les étudiants définissent-ils la recherche documentaire ? Dans quelle mesure et pourquoi fréquentent-ils les bibliothèques universitaires (BU) ? Pour quelles raisons effectuent-ils des recherches documentaires ? Et quelles sont les méthodes utilisées pour les recherches documentaires?

\section{CONTEXTES DE LA RECHERCHE DOCUMENTAIRE}

Les étudiants définissent la recherche documentaire de multiples manières : pour $79 \%$ c'est une sélection d'informations pertinentes et $63 \%$ considèrent qu'il s'agit de faire une liste de documents appropriés. $67 \%$ des étudiants estiment encore que la recherche documentaire s'accompagne d'une consultation de livres. Enfin, seulement $36 \%$ des étudiants lient la recherche documentaire au fait de se rendre dans une bibliothèque et $18 \%$ à l'emprunt des livres. La recherche documentaire n'est ainsi pas seulement associée à la bibliothèque et à ses services traditionnels.

En décembre, la quasi-totalité déclare fréquenter les BU (seuls 12 étudiants sur 174 ne le font pas). Dans notre enquête, la comparaison des déclarations des 
étudiants sur leurs pratiques à l'université et au lycée permet de faire émerger plusieurs constats. La BU rend différents services aux étudiants, mais dans des proportions moins importantes comparativement au CDI du lycée : photocopies (20\% contre $29 \%$ ), consultations d'Internet (31\% contre $55 \%$ ), rencontres des amis (13\% contre $24 \%)$. Ceci n'est guère étonnant dans la mesure où l'université offre d'autres espaces aux étudiants pour ces différents aspects de la vie étudiante parfois inexistants au lycée. Reste toutefois que $37 \%$ des étudiants fréquentant la BU y viennent pour travailler en groupe. La bibliothèque représente ainsi un lieu de travail pour les étudiants (Maresca et al., 2005).

Et plus encore, l'entrée à l'université se traduit par un changement des habitudes des jeunes: la proportion y travaillant leurs cours est plus forte $(85 \%$, soit + 29 points). La fréquentation de la bibliothèque pour le prêt d'ouvrages est légèrement en baisse (51 \%, soit moins 9 points), mais la consultation de documents sur place (presse ou ouvrages) est en hausse ( $67 \%$, soit +27 points). Il est cependant à noter que seulement $35 \%$ fréquentant la BU viennent chercher un ou plusieurs documents précis dont ils ont la référence et $23 \%$ cherchent une information précise sans avoir de référence précise au départ.

Pour 83 \% étudiants, la fréquentation du CDI au lycée était liée à la préparation des devoirs ou des exposés. La situation est différente lors de l'entrée à l'université dans la mesure où, à la fin du premier semestre d'enseignement, seulement un tiers des étudiants déclare avoir réalisé des recherches documentaires précises pour un travail demandé par un(e) enseignant(e) et seulement près de sept étudiants sur dix déclarent avoir fait des recherches documentaires afin de compléter leurs cours. Ceci renvoie aux principales motivations à la réalisation de recherches documentaires : 68 \% des étudiants déclarent les faire afin de compléter un cours et $59 \%$ pour préparer un exposé ou un devoir demandé par un enseignant, mais seulement $30 \%$ affirment que ces recherches sont liées à leur propre culture et information.

En général, pour travailler leurs cours, plus de la moitié des étudiants déclarent utiliser leurs notes de cours et compléter les enseignements dispensés par des lectures recommandées par les enseignants. Seulement $15 \%$ effectuent d'autres lectures. Ceci renvoie aux constats de Després-Lonnet et Courtecuisse (2006) sur l'importance attachée aux références bibliographiques données par les enseignants et à la valeur prescriptive de ces listes de références; cet attachement étant lié à des questions de temps et de complications engendrées par une démarche exploratoire. En revanche, seulement $43 \%$ déclarent faire des recherches complémentaires sur Internet et seul le tiers des étudiants fait des recherches à la bibliothèque pour travailler ses cours. Cette faible importance de la bibliothèque dans les stratégies de recherche documentaire des étudiants 
peut être liée à la tendance plus importante des étudiants de $1^{\text {re }}$ année à se limiter aux ressources dont ils disposent chez eux (Maresca et al., 2005).

\section{OUTILS DE LA RECHERCHE DOCUMENTAIRE}

Cette faible importance de la bibliothèque peut être reliée aux outils privilégiés par les étudiants pour leur travail comme pour leurs recherches documentaires. Ainsi, pour leur travail d'étudiant, les étudiants consultent différents outils, mais privilégient massivement Internet (75\%) et/ou un dictionnaire (papier ou en ligne) (70\%), puis des livres à $51 \%$ et des encyclopédies (papier ou en ligne) à $49 \%$ et des livres. Les revues spécialisées et la presse sont en revanche peu utilisées (respectivement 13 et $6 \%$ ). Concernant les recherches documentaires nécessaires à leur travail d'étudiant, $65 \%$ des étudiants utilisent Google ${ }^{\circledR}$ ou un autre moteur de recherche généraliste. Seul un tiers consulte les bases de données de la BU et seul un étudiant sur dix a le réflexe de se rendre à la $B U$. Précisons qu'en décembre, près de deux étudiants sur dix n'avaient pas encore fait ce type de recherches. Cette prédominance d'Internet est encore plus forte lorsqu'il s'agit de préparer un devoir ou un dossier, puisque, dans ce cas, près des trois quarts des étudiants recherchent des informations sur Internet. En fait, moins de la moitié des étudiants vont rechercher des ouvrages sur le sujet à la bibliothèque et seuls $40 \%$ se reportent à un ouvrage du type encyclopédie. Moins de trois étudiants sur dix se reportent à une bibliographie donnée par un(e) enseignant(e).

Parmi les outils disponibles sur Internet, les étudiants estiment que les plus utiles à leur travail sont les sites préconisés par les enseignants à $58 \%$ puis Wikipedia à $46 \%$. Pourtant, leurs pratiques se révèlent différentes : pour mener leurs recherches documentaires sur Internet, $93 \%$ des étudiants consultent des

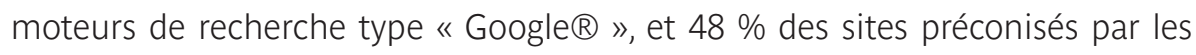
enseignants, contre seulement $13 \%$ des étudiants qui consultent des bases de données spécialisées ou les catalogues de bibliothèques. Seuls $13 \%$ utilisent le catalogue de la bibliothèque en se connectant à distance. On retrouve ici les constats de Candalot (2003) sur les représentations des entrants à l'université en matière de système d'information qui se réduisent le plus souvent à Internet. L'utilisation nettement plus restreinte des catalogues des bibliothèques ou d'autres bases de données serait ainsi notamment liée à leur méconnaissance de ces outils (Mittermeyer et Quirion, 2003) ; méconnaissance qui engendre alors des pratiques consistant à recourir spontanément à l'outil connu (Candalot, 2003). En outre, Internet est sans doute également privilégié en raison du sentiment de facilité et de liberté qu'il donne aux étudiants. C'est également un outil qui ne demande pas la maîtrise de plusieurs niveaux de connaissances 
et de compétences contrairement aux bases de données (Després-Lonnet et Courtecuisse, 2006). Le succès des outils d'écriture collaboratif devenus des ressources privilégiées s'explique sans doute en raison de la plus grande proximité de langage des auteurs et la simplicité de leur structure (Després-Lonnet et Courtecuisse, 2006).

Graphique 1. Les outils disponibles sur Internet utiles au travail universitaire préférés par les étudiants

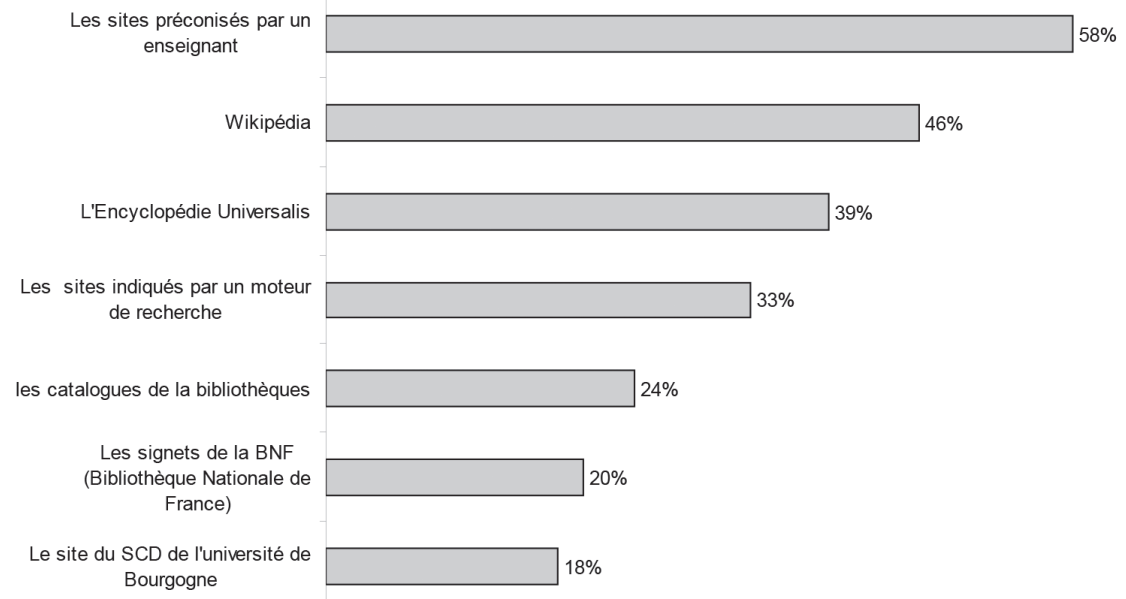

Note : ces indicateurs ont été construits en utilisant les 3 premiers outils mis en avant par les étudiants.

Nombre d'étudiants ne se posent pas la question de l'évaluation de la qualité de l'information trouvée sur Internet ou ne savent pas comment hiérarchiser ces informations (Poissenot, 2004). Dans notre enquête, deux tiers des étudiants déclarent « apprécier si les informations sont fiables ». Toutefois cette appréciation de la fiabilité reste discutable car, à titre d'exemple, seulement $43 \%$ regardent la date de mise à jour des sites et seulement un quart des étudiants se fie à la réputation du site (la notoriété des responsables ne fait pas de doute). En fait, les étudiants examinent ces informations collectées sur Internet d'une autre manière : la moitié examine si « les informations sont facilement exploitables », $33 \%$ regardent si « les informations du site sont variées » (documents interactifs, nombreux liens), $27 \%$ évaluent si « les informations sont suffisamment exhaustives » et $20 \%$ observent si « les informations sont faciles d'accès ». Les résultats de notre enquête rappellent les difficultés des étudiants à apprécier la pertinence de ces informations collectées sur Internet (Després-Lonnet et Courtecuisse, 2006).

Lorsque les étudiants utilisent un moteur de recherche généraliste comme Google $®$, plus de huit sur dix tapent les mots de leurs recherches. Seul un quart 
utilise la recherche avancée du moteur de recherche. Ces résultats de notre recherche renvoient aux constats établis par Després-Lonnet et Courtecuisse (2006) sur les modes de recherche des entrants à l'université avec Google ${ }^{\circledR}$. Pour rechercher un document à la BU, seulement $59 \%$ des étudiants cherchent l'ouvrage dans le catalogue informatisé, $49 \%$ parcourent les rayons. En fait, se cachent sans doute les difficultés des étudiants à utiliser les catalogues des bibliothèques en raison de leur manque de compétences, ainsi que l'ont montré Després-Lonnet et Courtecuisse (2006). Seulement $16 \%$ demandent l'aide d'un(e) bibliothécaire, ce qui correspond au bilan de Courtecuisse (2008) pour des étudiants en histoire.

Malgré la forte fréquentation du CDI au lycée (90\%), seulement $44 \%$ des étudiants déclarent avoir bénéficié d'une formation à la recherche documentaire au lycée. Toutefois, ils sont globalement satisfaits de la manière dont ils effectuent leurs recherches: seuls $11 \%$ ne s'en déclarent pas satisfaits. Durant la semaine d'intégration à I'UB, la quasi-totalité des étudiants ont visité une BU (96\%). Et $80 \%$ déclarent avoir assisté au cours de présentation du SCD.

\section{DIFFÉRENTES PRATIQUES DOCUMENTAIRES ÉTUDIANTES}

Cette recherche veut appréhender les différents types de pratiques étudiantes en termes de recherche documentaire, tout en sachant que certaines dimensions sont fortement interdépendantes. Pour ce faire, dans cette perspective empirique, les réponses des étudiants collectées dans le questionnaire ont été étudiées avec une analyse factorielle des correspondances multiples suivie d'une classification ascendante hiérarchique, afin de déterminer les différents groupes d'étudiants existants. Trois pratiques documentaires ont ainsi été dégagées. Les deux premières se singularisent par la fréquentation de la BU lors du premier semestre, alors que le troisième regroupe des étudiants n'ayant pas forcément pris cette habitude.

Le premier profil concerne près du tiers de la promotion (32\%). II regroupe des étudiants ayant été à la BU au cours du premier semestre pour emprunter ou rapporter des documents ou les consulter sur place comme pour faire des recherches de documents sans forcément avoir au préalable une référence précise. Plus que les autres, ceux-ci savent recourir au catalogue informatisé de la BU et peuvent y venir pour utiliser Internet. Durant le semestre, ils ont non seulement fait des recherches documentaires pour compléter leurs cours, mais aussi pour préparer un devoir pour un exposé demandé par un 
enseignant. Recourant comme les autres à Internet, ils ont plus tendance à utiliser des sites dont les informations sont variées et qu'ils estiment fiables. Plus que les autres, leurs recherches sur Internet peuvent être faites en s'appuyant sur les différentes fonctionnalités des moteurs de recherche. Ces étudiants peuvent être considérés comme « les plus expérimentés » au regard de leurs méthodes documentaires.

Le deuxième type concerne près de la moitié des étudiants (46\%). La recherche documentaire est plus appréhendée par le fait de sélectionner des informations pertinentes, que par le fait d'emprunter ou consulter des documents. Bien qu'ils se rendent à la $\mathrm{BU}$, ce n'est pas principalement pour mener des recherches documentaires. En effet, ils viennent pour y travailler en groupe, et ils recourent peu aux services dédiés à la recherche documentaire (emprunt de documents, utilisation de postes Internet à disposition). Comme le premier groupe, ils mènent des recherches documentaires afin de compléter leurs cours, mais les utilisent moins dans le cadre d'un devoir ou d'un exposé demandé par un enseignant. Leurs recherches documentaires s'appuient exclusivement sur la mobilisation d'un moteur de recherche comme Google ${ }^{\circledR}$, en ignorant les bases de données spécialisées ou les catalogues des BU. Ils complètent ainsi leurs cours en utilisant seulement des informations disponibles sur Internet et recourent peu à des ouvrages.

Enfin, regroupant $22 \%$ des étudiants, un troisième profil correspond aux étudiants qui mènent peu de recherches documentaires, même pour compléter leurs cours. Peu enclins à réaliser de telles recherches, ils n'y ont en outre pas été incités durant ce premier semestre, car ils n'ont pas eu de travaux demandés par les enseignants qui auraient pu être des facteurs de motivation. Menant rarement des recherches documentaires dans le cadre de leur travail d'étudiant, ils mobilisent ainsi peu Internet dans cet objectif et ne se rendent pas à la BU. Ils ne recourent donc pas aux services traditionnels offerts par les BU : ils n'ont ainsi ni recherché, ni consulté des documents et encore moins utilisé le catalogue informatisé.

Les sollicitations des enseignants paraissent importantes. En effet, les étudiants ayant eu des devoirs ou travaux demandés par les enseignants sont plus enclins à appartenir au groupe des étudiants dits « expérimentés ». Ce rôle des enseignants rejoint les conclusions de Maresca et al. (2005) concernant la fréquentation des bibliothèques par les étudiants de $1^{\text {re }}$ année. Mais ce rôle des enseignants pose également question, car une partie des étudiants déclare ne pas avoir eu à faire des devoirs demandés par les enseignants. Est-ce la conséquence de l'organisation des enseignements ou faut-il y voir un effet d'une plus faible implication dans les études? Retenons que l'utilisation d'Internet 
pour les études et la fréquentation de la BU sont complémentaires comme l'ont montré des recherches sur les publics des bibliothèques (Maresca, 2007).

\section{LES MÉTHODES DOCUMENTAIRES AU REGARD DES PROFILS ÉTUDIANTS}

Notre recherche montre que les styles documentaires sont différents selon les parcours étudiants. Ainsi, les bacheliers entrant sont proportionnellement moins nombreux parmi les étudiants dits « expérimentés », représentant seulement $65 \%$ des effectifs. En revanche, ces nouveaux bacheliers sont plus fréquemment des étudiants peu enclins à faire des recherches documentaires : ils représentent $82 \%$ de cette population, contre $73 \%$ des inscrits en $1^{\text {re }}$ année de LLCE à I'UB. Les redoublants se singularisent par le fait d'être surreprésentés parmi le groupe des « étudiants dits expérimentés » : $14 \%$ des effectifs contre $7 \%$ des inscrits. Enfin, les étudiants en situation de réorientation appartiennent proportionnellement moins au groupe peu enclin à faire des recherches documentaires : $15 \%$ des effectifs contre $21 \%$ des effectifs des autres groupes.

Au-delà de l'expérience à l'université, le baccalauréat est également discriminant. Les étudiants ayant obtenu leur baccalauréat avec mention (AB, B, TB) sont proportionnellement plus présents parmi les « expérimentés ». Ils représentent en effet $45 \%$ des effectifs, contre $38 \%$ des effectifs des deux autres groupes. En revanche, le type de baccalauréat n'est pas discriminant.

II n'existe pas de différence selon l'origine sociale. En revanche, il existe des différences hommes/femmes. La proportion d'hommes est plus importante parmi les « peu enclins à mener des recherches documentaires » : $28 \%$ contre $20 \%$ pour le groupe des « expérimentés » et $11 \%$ des étudiants « s’appuyant exclusivement sur Google ${ }^{\circledR} »$.

Une régression logistique multinomiale spécifiant les déterminants d’appartenance aux trois styles documentaires « toutes choses égales par ailleurs $»^{2}$, permet de mesurer le poids des différents facteurs. Le seul aspect discriminant est le sexe: les hommes ont plus de chances, que les femmes d'appartenir au groupe des étudiants « peu enclins à faire des recherches documentaires » qu'au groupe des étudiants « mobilisant exclusivement Google $₫$ » ou même au groupe des étudiants dits « expérimentés en termes de recherche documentaire ». L'appartenance aux deux groupes effectuant des recherches documentaires n'est pas marquée par des différences hommes-femmes. En fait, cette différence entre les

2. Étude réalisée pour 134 personnes, soit $77 \%$ des répondants (46 \% des inscriptions pédagogiques) compte tenu des problèmes d'authentification. 
filles et les garçons renvoie aux différences traditionnellement mises en avant par les travaux sur les étudiants : les étudiantes étant plus studieuses (Lahire, 1997).

\section{LA RÉUSSITE AU REGARD DES MÉTHODES DOCUMENTAIRES}

Ici, notre objectif est de rechercher si les différentes méthodes documentaires des étudiants peuvent être déterminantes pour la réussite. Cette étude s’appuie sur une analyse des différentes situations à l'issue de la $1^{\text {re }}$ session d'examens à partir d'une nomenclature en trois catégories: les étudiants admis aux examens, les étudiants ajournés et les étudiants défaillants. Les notes ont été collectées à partir des informations recensées dans Apogée 3 . Précisons que dans notre échantillon, la proportion d'étudiants admis s'élève à $59 \%$ au premier semestre, $40 \%$ ont été ajournés et $1 \%$ sont défaillants. Ces proportions ne reflètent pas les taux de réussite, puisque seuls les répondants au questionnaire sont inclus.

Des différences significatives existent entre les étudiants admis (moyenne supérieure ou égale à 10/20) et les ajournés (moyenne inférieure à 10/20). Ainsi, les étudiants définissant la recherche documentaire par le fait de faire une liste de documents appropriés échouent plus souvent que les autres : $56 \%$ ont une moyenne inférieure à 10/20 contre $37 \%$ des autres. Ce constat est également valable pour ceux considérant que la recherche documentaire est synonyme d'emprunter des livres: $51 \%$ n'obtiennent pas la moyenne aux examens contre 35 \% n'ayant pas une telle définition. Au-delà, il apparaît que les étudiants ayant I'habitude de solliciter les enseignants réussissent plus fréquemment leur premier semestre: $89 \%$ contre $56 \%$ des étudiants n'ayant pas ce type de démarche. Enfin, ceux qui se fient à la réputation des sites pour juger les informations trouvées sur Internet réussissent plus fréquemment leur premier semestre que les autres (72\% contre $54 \%$ ). Ces premiers constats doivent être examinés avec prudence car corrélation n'est pas synonyme de causalité.

Les étudiants peu enclins à mener des recherches documentaires échouent plus fréquemment aux examens (52\% contre respectivement 40 et $38 \%$ ). Les autres sont en revanche plus en situation de réussite à l'issue du premier semestre. (cf. tableau 1). De tels résultats renvoient aux travaux de Coulon (1999) faisant le lien entre réussite en $1^{\text {re }}$ année et maîtrise de la recherche documentaire.

3. Étude réalisée pour 129 personnes, soit $74 \%$ des répondants (44\% des inscriptions pédagogiques) compte tenu des problèmes d'authentification et des faibles effectifs défaillants. 
Tableau 1 : Résultats aux examens du premier semestre selon les pratiques documentaires

\begin{tabular}{|l|c|c|c|c|}
\hline \multicolumn{1}{|c|}{ Groupes } & Admis & Ajournés & Défaillants & Ensemble \\
\hline Expérimentés & 60 & 37 & 3 & $100 \%$ \\
\hline Mobilisant exclusivement sur Google $^{\circledR}$ & 62 & 38 & 0 & $100 \%$ \\
\hline $\begin{array}{l}\text { Peu enclins à faire des recherches } \\
\text { documentaires }\end{array}$ & 48 & 52 & 0 & $100 \%$ \\
\hline
\end{tabular}

Toutefois, des précautions doivent être prises pour interpréter ces résultats. En effet, la réussite doit être examinée en prenant compte les styles documentaires mais en intégrant également d'autres facteurs de la réussite. À cet effet, l'analyse de la réussite versus l'échec au premier semestre (apprécié par une moyenne générale inférieure à 10/20) réalisée à partir d'une régression logistique ${ }^{4}$ (dont l'intérêt est d'estimer le poids de chaque facteur sur la réussite) permet d'avancer des résultats : les étudiants ayant obtenu une mention au baccalauréat ou titulaires d'un baccalauréat général, comme les redoublants, sont moins souvent ajournés aux examens du premier semestre. Et, la probabilité de réussite des enfants de cadres est plus forte. Nos résultats sont conformes aux principales conclusions sur la réussite à l'entrée à l'université.

En revanche, cette régression montre qu'il n'y a pas de différences significatives en termes de réussite en fonction des pratiques documentaires adoptées par les étudiants depuis le début de l'année, ni en fonction du fait d'avoir ou non assisté à la formation réalisée par le SCD. Ces résultats ne viennent pas directement contredire les travaux antérieurs. Ils renvoient en fait à un problème exploré par Coulon se demandant si les carrières différentes des étudiants selon qu'ils aient ou non suivi un enseignement à la recherche documentaire n'était pas en fait le reflet que celui-ci pouvait être plus massivement choisi par « des étudiants plus favorisés socioculturellement et mieux informés parce qu'ils en connaissaient ou devinaient l'efficacité formatrice » (Coulon, 1999, p. 38). Dans notre recherche, les effets non significatifs des pratiques documentaires renvoient au fait que ces derniers sont plus fréquemment associés à des étudiants particuliers ayant les plus fortes chances de réussite, c'est-à-dire redoublants et les jeunes ayant une mention au baccalauréat (AB, B et TB). À notre connaissance, cette dernière dimension du passé scolaire n’avait pas été explorée dans les travaux sur les effets de la recherche documentaire. Malheureusement, notre échantillon ne permet pas une telle étude.

4. Au premier semestre, l’analyse des défaillants est impossible compte tenu des effectifs. 


\section{DISCUSSION}

Rappelant le poids d'Internet dans les pratiques des entrants à l'université, cette recherche révèle l'existence de modalités différenciées en termes de recherche documentaire: coexistent trois types d'étudiants ayant des habitudes documentaires différentes pour leurs études. L'hétérogénéité des habitudes de travail au sein d'une formation est ainsi redite (Trinquier et al., 1999). Si les bacheliers de l'année sont proportionnellement moins nombreux parmi les plus expérimentés et plus nombreux parmi ceux n'effectuant pas de recherches documentaires pour leurs études, le parcours scolaire n'est pas un élément d'explication des pratiques documentaires adoptées. Seule persiste une différence entre hommes et femmes.

L'usage d'Internet est complémentaire de la fréquentation de la BU selon des modalités différentes. Ainsi les « experts » associent Internet et le recours aux services traditionnels de documentation des bibliothèques (utilisation du catalogue, emprunts et consultation d'ouvrages), les autres mobilisant exclusivement Google ${ }^{\circledR}$ viennent le plus souvent en bibliothèque pour travailler en groupe. Les BU ne sont pas seulement des lieux de recherche d'informations: lieux de travail (Maresca et al., 2005 ; Dufils, 2010), elles sont désormais investies pour travailler en groupe.

Les sollicitations des enseignants sont importantes pour impulser une démarche de recherche documentaire chez les entrants à l'université. Toutefois, ces sollicitations ne touchent pas l'ensemble des étudiants d'une même promotion. Il est difficile de déterminer si ceci est la conséquence de l'organisation des enseignements ou d'une plus faible implication dans les études. Appréhender conjointement les habitudes de travail, les méthodes documentaires et les motivations étudiantes au regard de l'organisation des enseignements est donc nécessaire pour lever cette limite de notre recherche.

Conforme aux bilans sur la pauvreté des compétences en termes de maîtrise de l'information à l'entrée à l'université (Mittermeyer et Quirion, 2003; Thirion et Pochet, 2008), cette recherche rappelle les difficultés des étudiants concernant la recherche et la sélection des informations sur Internet (Després-Lonnet et Courtecuisse, 2006), alors qu'ils sont plutôt satisfaits de la manière dont ils effectuent leurs recherches. Ce décalage ne peut être qu'un obstacle à l'adhésion des étudiants à des programmes de formation documentaire promus dès la fin des années quatre-vingt-dix et renouvelés par certaines universités françaises dans le cadre du Plan réussite en licence (PRL). Ce décalage risque en effet de compromettre ces formations en renvoyant aux constats faits sur d'autres dispositifs pédagogiques (Danner, 2000 ; Borras 2011). D’autant que seuls $40 \%$ 
des étudiants interrogés dans cette recherche estiment qu'une formation à la recherche documentaire pourrait les aider.

Thirion (2003) a rappelé la rareté des travaux sur les effets de la maîtrise de l'information et leur nécessité pour promouvoir les formations des professionnels de la documentation. Avec une démarche plus large que l'étude des effets de la fréquentation des BU (Gruel, 2002), notre recherche souligne que la réussite est corrélée avec différentes pratiques documentaires associées aux études:

- les jeunes ayant une démarche de recherche d'information sont proportionnellement plus nombreux à réussir leur premier semestre à l'image des résultats de Coulon (1999);

- la réussite étant plus importante avec des démarches expérimentées. Ceci remet en avant le poids des compétences techniques en termes de maîtrise de l'information; les « experts » ayant plus fréquemment recours à différents outils et aux fonctionnalités plus complexes des outils offerts notamment sur Internet.

Toutefois, l'intégration d'autres facteurs explicatifs de la réussite n'affirme pas le rôle déterminant de cette pratique; celle-ci étant portée par des étudiants ayant les plus fortes chances de réussite par ailleurs. Nos travaux permettent difficilement de distinguer le poids du passé scolaire de celui des pratiques documentaires. Cette limite importante ne pourra être dépassée que par d’autres études portant sur de plus grand nombre d'étudiants et des contextes différents ciblant les profils des étudiants.

\section{Cathy Perret}

Centre d'innovation pédagogique et d'évaluation (CIPE) Institut de recherche sur l'éducation (IREDU-CNRS) - Université de Bourgogne

\section{Bibliographie}

Boyer R., Coridian C. (2002). Réapprendre à apprendre pour réussir sa première année de DEUG. L'Orientation scolaire et professionnelle, vol. 31, n 3, p. 429-452.

Bertrand A.-M. (2002). À quoi sert l'Internet à l'université ? Bulletin des bibliothèques de France, $n^{\circ} 3$, http://bbf.enssib.fr/consulter/bbf-2002-03-0083-003 (consultation: 10 janvier 2012).

Borras I. (2011). Le tutorat à I'université : peut-on forcer les étudiants à la réussite ? Bref, $\mathrm{n}^{\circ} 290.4 \mathrm{p}$.

Candalot C. (2003). La formation aux compétences informationnelle à l'université : une voie ouverte pour le développement des sciences de l'information et de la communication. 
Xe Colloque bilatéral franco-roumain, CIFSIC Université de Bucarest, 28 juin - 3 juillet 2003 [Document en ligne], http://archivesic.ccsd.cnrs.fr/docs/00/06/22/25/PDF/sic_00000586. pdf (consulté le 14 avril 2012)

Coulangeon P. (2009). Que sont les héritiers devenus ? Les loisirs et les pratiques culturelles des étudiants en France. In L. Gruel, O. Galland, G. Houzel (dir.). Les étudiants en France: histoire et sociologie d'une nouvelle jeunesse. Rennes: Presses Universitaires de Rennes, p. 291-306.

Courtecuisse J.-F. (2008). La complémentarité « livres - Internet » dans les pratiques documentaires des étudiants en histoire. Document de travail, http://archivesic.ccsd.cnrs.fr/ docs/00/34/87/20/PDF/JF_Courtecuisse_-_Texte_complet.pdf (consultation : 10 janvier 2012).

Coulon A. (1997). Le métier d'étudiant: I'entrée dans la vie universitaire. Paris: Presses universitaires de France.

Coulon A. (1999). Un instrument d'affiliation intellectuelle : I'enseignement de la méthodologie documentaire dans les premiers cycles universitaires. Bulletin de Bibliothèques de France, vol. 44, n 1 , p. 36-42.

Danner M. (2000). À qui profite le tutorat mis en place dans le premier cycle universitaire? Les Sciences de l'éducation - Pour l'Ère nouvelle, vol. 33, n 1, p. 25-41.

Després-Lonnet M., Courtecuisse J.-F. (2006). Les étudiants et la documentation électronique. Bulletin de Bibliothèques de France, vol. 51, $n^{\circ}$ 2, p. 33-41.

Dubet $F$. (1994). Dimensions et figures de l'expérience étudiante dans l'université de masse. Revue française de sociologie, vol. $35, n^{\circ} 4$, p. 511-532.

Dufils É. (2010). Enquête auprès des usagers de la bibliothèque universitaire de Paris 8. Bulletin des bibliothèques de France, n 5, p. 36-39, http://bbf.enssib.fr/consulter/bbf-201005-0036-006 (consultation : 10 janvier 2012).

Faurie I., Almudever B., Hajjar V. (2004). Les usages d'internet des étudiants : facteurs affectant l'intensité, l'orientation et la signification des pratiques. L'Orientation scolaire et professionnelle, vol. 33, n³, p. 429-452.

Faurie I., Van de Leemput C. (2007). Influence du sentiment d'efficacité informatique sur les usages d'Internet par les étudiants. L'orientation scolaire et professionnelle, vol. $36, n^{\circ} 4$, p. 533-552.

Frenette M., Johnsson-Smaragdi U. (2004). A cross-national study on university students' experiences with the Internet in Shaping the Future of Communication research in Europe. ECA European Communication Association, Istanbul, April. http://edutice.archivesouvertes.fr/docs/00/03/40/72/PDF/Frenette.pdf consultation : 11 juin 2012).

Galland O. (2009). Autonomie, intégration et réussite universitaire ». In L. Gruel, O. Galland et G. Houzel (dir.). Les étudiants en France: histoire et sociologie d'une nouvelle jeunesse. Rennes: Presses universitaires de Rennes, p. 349-380.

Gruel L. (2002). Les conditions de réussite dans l'enseignement supérieur. OVE Infos, $n^{\circ} 2$, p. 1-10. 


\section{PRATIQUES DE RECHERCHE DOCUMENTAIRE ET RÉUSSITE UNIVERSITAIRE}

Jones S. (dir.) (2002). The Internet goes to colleges: how students are living in the future with to day's technology. Pew Research Centre, http://www.pewinternet.org/ /media/Files/ Reports/2002/PIP_College_Report.pdf.pdf (consultation : 17 janvier 2012).

Lahire B. (1997). Les manières d'étudier. Paris : La documentation française.

Lima L. Granon A. (2007). Les étudiants grenoblois et Internet: almanach des données: enquête 2006-2007. Grenoble: Grenoble Universités; LSE UPMF Grenoble.

Oberti M. (1995). Les étudiants et leurs études. In O. Galland (éd.). Le monde des étudiants. Paris: PUF, p. 23-54.

Maresca B. (2007). Les bibliothèques municipales en France après le tournant Internet: attractivité, fréquentation et devenir. Paris : Bibliothèque publique d'information; Centre Pompidou.

Maresca B., Dupuy C., Cazenave A. (2005). Enquête sur les pratiques documentaires des étudiants, chercheurs et enseignants-chercheurs de l'Université Pierre et Marie Curie (Paris 6) et de l'Université Denis Diderot (Paris 7). Paris : Crédoc, http://www.credoc.fr/pdf/ Rapp/R238.pdf (consultation : 6 janvier 2012).

Mittermeyer D., Quirion D. (2003). Étude sur les connaissances en recherche documentaire des étudiants entrant au premier cycle dans les universités québécoises. Montréal : Conférence des recteurs et des principaux des universités du Québec.

Poissenot C. (2004). La fin des héritiers: lecture et compétence documentaire des étudiants d'aujourd'hui. Présenté à la Journée d'études « Les étudiants et la lecture », Arras, Université d'Artois, 16 mars. [document électronique]. En ligne sur http://hal.archives-ouvertes. fr/docs/00/06/23/65/HTML/ (page consultée le 25 février 2013)

Renoult D. (2006). Enquêtes de publics dans les bibliothèques universitaires: où en sommes-nous ? Bulletin des bibliothèques de France, vol. 51, $n^{\circ} 2$, Consulté sur http://bbf. enssib.fr/consulter/bbf-2006-02-0005-001 (consultation : 10 janvier 2012).

Thirion P. (2003). L'impact des formations à la maîtrise de l'information sur la performance scolaire des étudiants : de la conviction à la preuve ? Présenté aux Journées FORMIST 2003 «Formation à la maîtrise de l'information à l'heure de l'harmonisation européenne: problématiques et perspectives », Enssib, Villeurbanne. [document électronique]. En ligne sur http://www.enssib.fr/bibliotheque-numerique/document-1863 (page consultée le 25 février 2013).

Thirion P., Pochet B. (dir.) (2008). Enquête sur les compétences documentaires et informationnelles des étudiants qui accèdent à l'enseignement supérieur en Communauté française de Belgique. Bruxelles : Conseil Interuniversitaire de la Communauté française de Belgique [CIUF]; EduDOC.

Trinquier M.-P., Clanet J., Alava S. (1999). Hétérogénéité et réussite dans le premier cycle universitaire: conditions perçues et effectives des pratiques d'études et d'enseignement. Paris: INRP; CNCRE. 


\section{Annexe: le questionnaire}

\begin{tabular}{|c|c|}
\hline I. Pour mieux vous situer : & II. Vos méthodes de travail \\
\hline 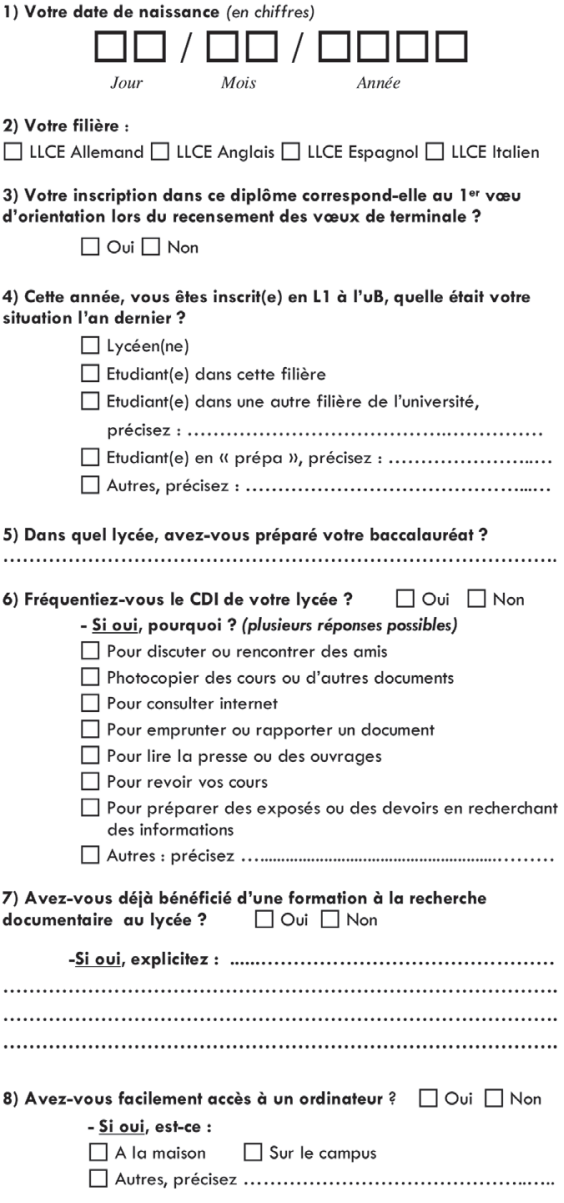 & 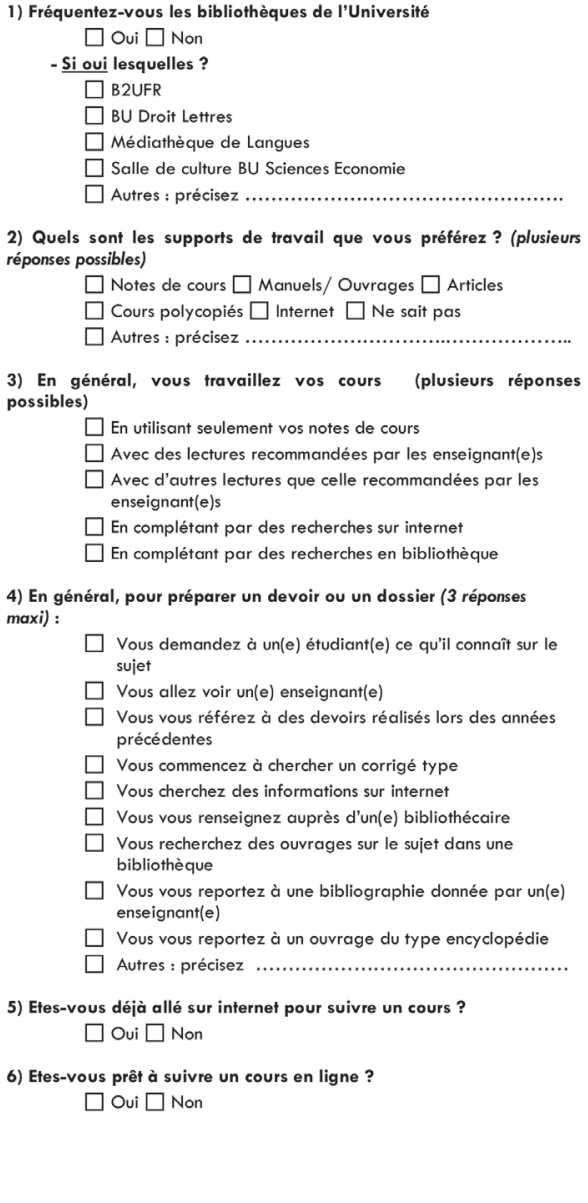 \\
\hline
\end{tabular}




\section{La recherche documentaire et vous}

1) Quelle définition donnez-vous pour " recherche documentaire "? (plusieurs réponses possibles)

$\square$ Emprunter des livres

$\square$ Consulter des livres

$\square$ Sélectionner des informations pertinentes

$\square$ Faire une liste de documents appropriés

$\square$ Aller à la Bibliothèque

2) Que faites-vous à la bibliothèque universitaire ? (plusieurs réponses possibles)
$\square$ Vous n'allez jamais à la bibliothèque universitaire
$\square$ Vous discutez et rencontrez vos amis
$\square$ Vous faites des photocopies
$\square$ Vous empruntez et rapportez des documents
$\square$ Vous consultez des documents sur place
$\square$ Vous travaillez sur vos cours
$\square$ Vous travaillez en groupe
$\square$ Vous cherchez un ou plusieurs documents précis dont vous avez les références
$\square$ Vous cherchez une information précise sans avoir de référence précise au départ
$\square$ Vous consultez interne

\section{3) Comment cherchez-vous un ouvrage à la}
bibliothèque universitaire ? (plusieurs réponses possibles)
$\square$ Vous ne savez pas
$\square$ Vous n'êtes pas encore allé(e) chercher d'ouvrages
$\square$ Vous demandez au (à la) bibliothécaire
$\square$ Vous cherchez l'ouvrage dans le catalogue informatisé de la bibliothèque
$\square$ Vous parcourez les rayons
$\square$ Autres : précisez .......................................

4) Dans quel contexte, faites-vous des recherches documentaires ? (plusieurs réponses possibles)

$\square$ Pour votre propre culture et information

$\square$ Pour préparer un devoir ou un exposé demandé par un(e) enseignant(e)

$\square$ Pour compléter vos cours

$\square$ Vous ne faites jamais de recherche documentaire

$\square$ Autres : précisez............................................

5) Depuis le début de l'année, avez-vous fait des recherches documentaires précises?

- pour un travail demandé par un(e) enseignant(e)

$\square$ Oui $\square$ Non

- pour compléter vos cours : $\square$ Oui $\square$ Non

6) Comment faites-vous vos recherches documentaires pour votre travail d'éfudiant(e)?

$\square$ Vous n'avez pas fait de recherche documentaire jusqu'à présent

$\square$ Vous ne savez pas

$\square$ Vous vous rendez à la bibliothèque pour vous faire aider

$\square$ vous allez voir sur "Google " ou un autre moteur de recherche généraliste

$\square$ Vous consultez les bases de données de la bibliothèque universitaire.

7) Que faites-vous sur internet pour mener des recherches documentaires?

$\square$ Vous ne connaissez rien à Internet

$\square$ Vous consultez le catalogue de la bibliothèque en vous connectant à distance

$\square$ Vous utilisez seulement internet pour communiquer ou pour vos loisirs, etc.

$\square$ Vous consultez des moteurs de recherche type "Google "

$\square$ Vous consultez des bases de données spécialisées ou les catalogues de bibliothèques

$\square$ Vous consultez des sites préconisés

$\square$ Autres : précisez
8) Lors de vos recherches sur internet, comment jugez-vous la qualité des informations que vous y avez trouvées? (cochez 4 cases au maximum)

$\square$ Vous examinez si les informations sont facilement exploitables $\square$ Vous regardez si les informations du site sont variées (documents interactifs, nombreux liens)

$\square$ Vous évaluez si les informations sont suffisamment exhaustives

$\square$ Vous appréciez si les informations sont fiables

$\square$ Vous vous fiez à la réputation du site (la notoriété des responsables ne fait pas de doute)

$\square$ Vous regardez la date de mise à jour des sites

$\square$ Vous observez si les informations sont faciles d'accès

9) Etes vous satisfait de la manière dont vous faites des recherches documentaires?

$\square$ Très satisfait $\square$ Satisfait $\square$ Pas satisfait $\quad \square$ Pas du tout satisfait

10) Pensez-vous qu'une formation à la recherche documentaire puisse vous aider dans vos études?

$\square$ Tout à fait d'accord

$\square$ D'accord

$\square$ Pas d'accord

$\square$ Pas du tout d'accord

$\square$ Ne sait pas

11) Si vous utilisez un moteur de recherche généraliste comme "Google ", comment faites-vous vos recherches :

$\square$ Vous tapez les mots de votre recherche

$\square$ Vous utilisez la recherche avancée du moteur de recherche

12) Quels outils documentaires consultez-vous pour les recherches documentaires nécessaires à vołre travail d'éfudiant? (classez par ordre de préférence de 7 à 7 , si vous n'utilisez pas un des ses outils, ne le classez pas)

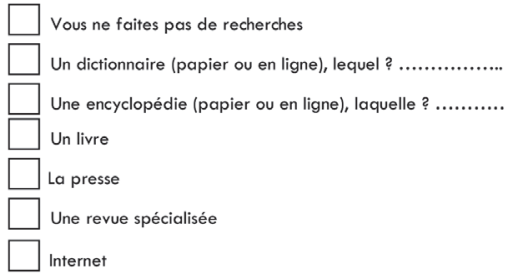

13) A votre avis, quels sont les outils disponibles sur internet utiles votre travail universitaire? (classer par ordre d'importance de 1 à 8 )

$\square$ Les sites indiqués par un moteur de recherche
$\square$ L'Encyclopédie Universalis
$\square$ Le site du SCD de l'université de Bourgogne
$\square$ Les signets de la BNF (Bibliothèque Nationale de France)
$\square$ Les catalogues de bibliothèque
$\square$ Wikipedia
$\square$ Les sites préconisés par un(e) enseignant(e)
$\square$ Autres : précisez .....................................................


IV. Votre perception de la formation à la recherche documentaire réalisée par le SCD (Service Commun de Documentation de l'université de Bourgogne)

1) Durant votre semaine d'intégration, avez-vous visité une bibliothèque de l'université ?

$\square$ Oui $\square$ Non $\square$ Ne sait pas

2) Durant votre semaine d'intégration, avez-vous assisté au cours de présentation du Service Commun de Documentation (SCD) de l'université de Bourgogne en cours?

$\square$ Oui $\square$ Non $\square$ Ne sait pas

- $\underline{\text { Si ovi, }}$

- Quelle est l'information principale que vous avez retenue?

(1)

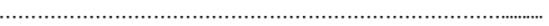

- Avez-vous eu des informations utiles?

$\square$ Oui $\square$ Non $\square$ Ne scit pas

- Les informations données sont : (plusieurs réponses possibles)

$\square$ Trop nombreuses
$\square$ Pas assez nombreuses
$\square$ Suffisantes
$\square$ Intéressantes
$\square$ Trop complexes
$\square$ Ne sait pas

- La durée de la présentation était

$\square$ Suffisante $\square$ Trop longue $\square$ Trop courte

- Grâce à cette présentation, pensez-vous être plus efficace dans vos recherches documentaires?

$\square$ Oui $\square$ Non $\square$ Ne sait pas

- La date de cette présentation vous paraît-elle appropriée?

$\square$ Oui $\square$ Non $\square$ Ne scit pas

- Si non, elle serait plus judicieuse

$\square$ A la fin du ler semestre (avant Noël)

$\square$ Au début du $2^{\text {nd }}$ semestre (février)

$\square$ Autres : précisez...

3) Parmi les services offerts aux étudiants par le SCD de l'université de Bourgogne quels sont ceux qui vous paraissent les plus utiles? (4 réponses possibles)

$\square$ Prêt de documents

$\square$ Prêt entre bibliothèques

$\square$ Consultation sur place de documents

$\square$ Accès Wifi

$\square$ Places de travail

$\square$ Bureau d'information

$\square$ Postes informatiques

$\square$ Photocopies

4) Avez-vous déjà consulté le site web du SCD ? $\square$ Oui $\square$ Non - $\underline{\text { Si oui, pourquoi? }}$

$\square$ Consulter les horaires d'ouverture

$\square$ Consulter votre compte de lecteur

$\square$ Connaître la Bibliothèque où se trouve la documentation

$\square$ Consulter la disponibilité d'un ouvrage ou d'un document

$\square$ Faire une recherche sur un thème précis

$\square$ Consulter la presse en ligne

$\square$ Consulter une base de données
5) Que trouve t-on dans une notice bibliographique ? (plusieurs réponses possibles)
$\square$ Un titre
$\square$ Une cote
$\square$ Une date d'édition
$\square$ Un auteur
$\square$ La localisation du documen
$\square$ Le nombre de pages
$\square$ L'image de la couverture

6) Quelles sont parmi les propositions suivantes celles qui correspondent à des titres de bases de données existantes (plusieurs réponses possibles.
$\square$ Généraliste
$\square$ CAIRN
$\square$ Francis
$\square$ François
$\square$ Vox
$\square$ Europresse

7) Documents primaires et secondaires : cochez la bonne case

\begin{tabular}{|c|c|c|}
\hline & $\begin{array}{l}\text { Document } \\
\text { primaire }\end{array}$ & $\begin{array}{l}\text { Document } \\
\text { secondaire }\end{array}$ \\
\hline Roman & & \\
\hline Base de données & & \\
\hline Dictionnaire & & \\
\hline Article de revue & & \\
\hline Catalogue & & \\
\hline $\begin{array}{c}\text { Bibliographie fournie } \\
\text { par l'enseignant }\end{array}$ & & \\
\hline
\end{tabular}

8) Pour aborder un sujet avec lequel vous n'êtes pas familier, vous consultez d'abord
$\square$ Une revue
$\square$ Le catalogue informatisé de la Bibliothèque Universitaire
$\square$ Une encyclopédie papier ou en ligne
$\square$ Une base de données
$\square$ Un livre
$\square$ Un moteur de recherche
$\square$ Ne sait pas

9) A votre avis, pour améliorer les recherches d'informations sur internet on peut : (3 réponses maximum)

$\square$ Utiliser des ressources conçues par des professionnels de la documentation (signets, portails)

$\square$ Déchiffrer l'adresse URL des sites

$\square$ Essayer d'évaluer la valeur des sites consultés

$\square$ Utiliser des moteurs de recherche généraliste

$\square$ Poser la question dans un forum

$\square$ Ne sait pas

$\square$ Autres : précisez 